$17^{\text {th }}$ International Congress of Metrology, 14006 (2015)

DOI: $10.1051 /$ metrology / 201514006

(C) Owned by the authors, published by EDP Sciences, 2015

\title{
Advances in measurement of interfacial chemical activity
}

\author{
Andrew J. Wain ${ }^{1}$ \\ ${ }^{1}$ National Physical Laboratory, Hampton Road, Teddington, TW11 OLW, United Kingdom
}

\begin{abstract}
The latest developments in interfacial mapping at NPL using scanning electrochemical microscopy (SECM) are reviewed. A suite of electrochemical imaging techniques has been developed that enable the multiscale mapping of electroactivity at solid-liquid interfaces, as demonstrated by a number of applications. Micronscale imaging reveals non-uniform activity distribution across model catalyst films and microarray screening provides a convenient approach to compare the performance of electrocatalyst and photoactive materials for emerging energy conversion technologies. Imaging at the nanoscale is achieved using hybrid techniques that combine SECM with atomic force microscopy (AFM) and scanning ion conductance microscopy (SICM). Advances are demonstrated by topographical-electrochemical mapping of graphene flakes and imaging oxygen reduction electroactivity at the single nanoparticle level.
\end{abstract}

\section{Introduction}

Interfacial processes are ubiquitous and play a central role in a wide range of applications. Interest in solidliquid and solid-gas interfaces ranges from highly active surfaces, for example in heterogeneous catalysis and energy conversion technologies, to chemically inert materials, such as corrosion-resistant alloys. In these and many other examples the development and optimisation of new functional materials relies on our ability to measure, understand, and ultimately control interfacial reactivity.

A wide range of characterisation techniques are available for routine chemical analysis of interfaces, but determining the chemical composition of a surface alone fails to reveal information about chemical process dynamics, particularly because such measurements are commonly undertaken in unreactive environments. In situ and in operando measurements are key to probing interfacial chemistry and a great deal of interest lies in the development of new characterisation tools for such applications. A major challenge lies in the spatial nonuniformity in interfacial activity that is inherent to so many materials and renders macroscopic/bulk measurements inadequate. Mapping techniques that enable the distribution of chemical reactivity across a surface to be visualised provide a powerful solution to this challenge.

A notable example is scanning electrochemical microscopy (SECM), which allows electrochemical mapping of solid-liquid interfaces by bringing a micro or nano-electrode probe in close proximity to the surface and performing localised potential or current measurement.[1] Significant advances have been made over the last decade in this field, both in terms of spatial resolution and the depth of information gleaned, and these developments have been reviewed elsewhere.[2-5] This paper highlights recent activities at NPL in the development of multiscale electrochemical imaging tools and their application to a wide range of materials and interfacial processes.

\section{Imaging at the micron scale}

Conventional SECM imaging involves immersing the sample of interest into an electrolyte solution and scanning a microelectrode probe in the $x$ - and $y$ directions across the surface, typically maintaining a constant $z$-position (height). Probes usually comprise a $1-25 \mu \mathrm{m}$ diameter platinum, gold or carbon wire encapsulated in glass and exposed at the tip to reveal a disk electrode surrounded by glass insulation. Amperometric SECM requires the presence of a redox active mediator dissolved in the electrolyte solution that is oxidized or reduced reversibly at the tip under mass transport control. Since the current registered at the tip depends on the local concentration of the mediator, it is sensitive not only to the tip-surface separation, but also the ability of the surface to restore electrolysed mediator molecules to their original oxidation state.

This basic operation of SECM is exemplified in Figure 1, which illustrates its application to mapping the activity of a Pt/C fuel cell anode catalyst film, reported by Nicholson et al.[6] In this case the redox mediator is the $\mathrm{H}^{+}+\mathrm{e}^{-} \rightarrow 1 / 2 \mathrm{H}_{2}$ redox couple. The sample is immersed into an acidic solution and protons are reduced electrochemically at the tip to generate molecular hydrogen at the limit of mass transport. If the tip is approached towards an inert sample, hindered diffusion of protons results in a drop in current (termed negative feedback). Conversely, if the tip is approached 
towards an active surface, in this case the Pt catalyst capable of oxidising hydrogen (as in the anode reaction of a hydrogen fuel cell), the local regeneration of protons leads to an augmented current at the tip (positive feedback). Scanning laterally across the surface at a working distance of $10 \mu \mathrm{m}$ generates an activity map of the sample, which exhibits clear heterogeneity at this scale, as expected. It is important to recognise that measurements performed at a constant height in this way may exhibit current variations due to the surface roughness. In this work it was demonstrated that the sample topography was relatively flat, indicating that the observed variations can be attributed to the non-uniform hydrogen oxidation activity across the surface, presumably resulting from the irregular distribution of the Pt catalyst.

(a)

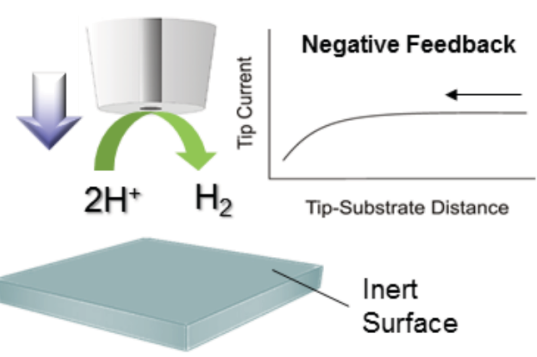

(b)

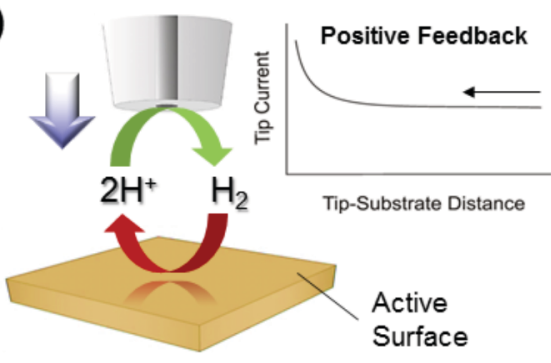

(c)

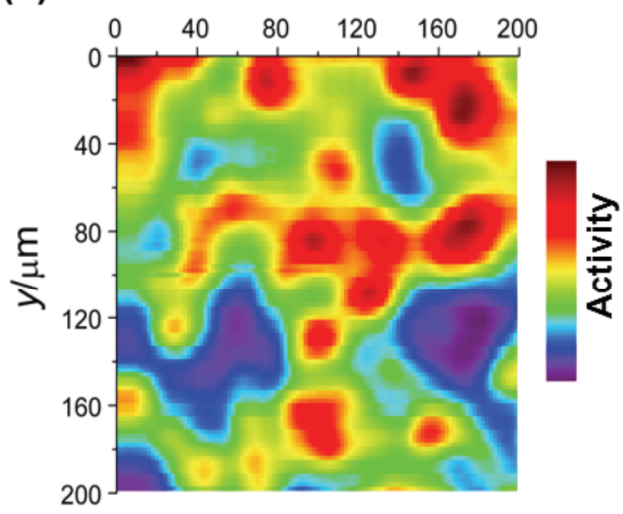

Figure 1. Basic operation of SECM depicting (a) negative feedback and (b) positive feedback for the proton reduction/hydrogen oxidation redox system. (c) SECM image of $\mathrm{Pt} / \mathrm{C}$ fuel cell catalyst film showing the distribution of hydrogen oxidation activity (adapted from [6]).

\section{SECM as a screening tool}

A useful application of SECM is in electrochemical materials screening. In this case the spatial element of the technique enables the performance of a range of materials immobilized on a single surface to be compared under identical conditions. A common approach is to use a microarray platform in which a library of different materials can be prepared in the form of 100 - $500 \mu \mathrm{m}$ spots.[7] A prominent example of SECM materials screening is to examine the activity of electrocatalyst arrays as a means to optimise variables such as composition and particle size. Oxygen reduction is a common target reaction due to its limiting role in fuel cell anodes.

A demonstration of this is shown in Figure 2, which depicts a microarray of gold nanoparticles immobilized on glassy carbon that has been mapped using SECM in the competitive oxygen reduction mode.[8] In this mode oxygen is reduced electrochemically at the tip as well at the surface, such that active electrocatalyst spots deplete the oxygen locally, which is registered as a decrease in tip current. In this array each of the spots consists of an ensemble of gold nanoparticles of a different average diameter, ranging from $5-50 \mathrm{~nm}$. Changing the substrate bias reveals that the smallest nanoparticles deplete the oxygen most effectively, indicating an increase in oxygen activity with decreasing particle diameter. This behaviour can be rationalized by the exposure of more active gold facets as the nanoparticles get smaller.

(a)

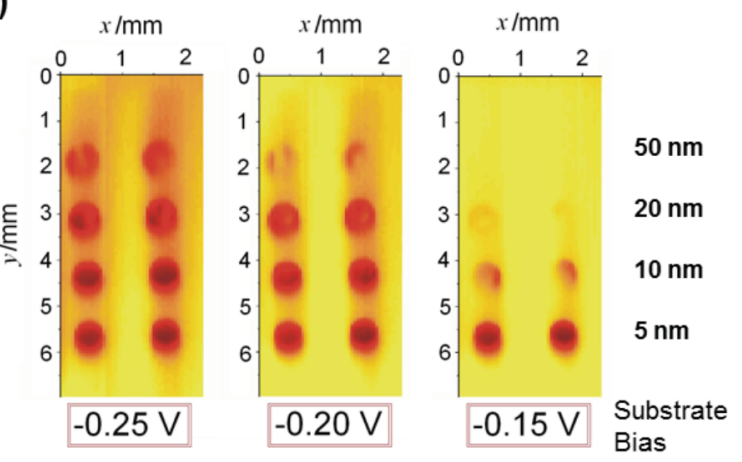

(b)

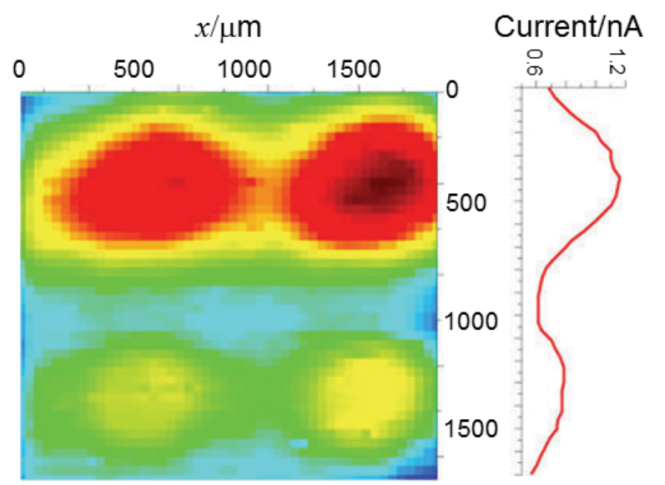

Figure 2. (a) Oxygen reduction activity screening of gold nanoparticles in the size range $5-50 \mathrm{~nm}$ immobilized as a microarray (adapted from [8]). (b) Photocurrent SECM map of N719 sensitizer array on $\mathrm{TiO}_{2}$, adsorbed at high (top row) and low (bottom row) dye coverages (adapted from [9]). 
This concept has been extended to the analysis of photocatalysts and other photoactive materials for energy conversion applications. Kylberg et al. used the iodide/triiodide redox couple to compare the photoactivity of different sensitizer materials, and different dye coverages adsorbed onto $\mathrm{TiO}_{2}$ surfaces under illumination.[9] Figure $2 \mathrm{~b}$ shows a $2 \times 2$ array of a common sensitizer, N719, adsorbed at two different coverages, which indicates that the method is sensitive to qualitative changes in photoactivity. The relative performances of different dyes were also found to be consistent with bulk solar cells testing, suggesting that this approach could be used for photoactive dye screening, potentially circumventing issues associated with repeatability in complete cell testing.

\section{Imaging at the nanoscale}

The SECM images presented thus far have spatial resolution in the tens of microns, but there is significant interest in performing electrochemical mapping at the nanoscale. There are two major challenges associated with undertaking high resolution SECM: (i) the probe electrode must have nanoscopic dimensions and (ii) the $z$-position of the probe must be tightly controlled at a short $(1-100 \mathrm{~nm})$ working distance from the surface. In most cases this calls for specialized probes that are not only small in size, but are integrated with a mechanism of positional feedback that allows the probe to track the surface closely during measurement. There are several approaches to this, which often involve combining SECM with existing topographical imaging microscopies. Unlike conventional SECM, these high resolution methods are not widespread and are limited to a relatively small number of groups. Our laboratory has focused on two of these methods, namely the combination of SECM with atomic force microscopy (AFM) and with scanning ion conductance microscopy (SICM), each of which will be discussed below.

\subsection{SECM-AFM}

The concept of combining SECM with AFM has been established for over a decade,[10] although widespread uptake of the approach has been impeded by challenges associated with fabricating specialised dual function probes. We have demonstrated SECM-AFM imaging with two different types of cantilever probe manufactured commercially, namely needle probes (Figure 3a)[11] and batch-fabricated conical probes (Figure 3b).[12] These probes enable topographicalelectrochemical imaging by use of "Lift Mode", in which each line of the image is scanned twice, first to collect the topography data in contact or tapping mode, and subsequently to record the electrochemical signal at a predefined lift height (e.g. $100 \mathrm{~nm}$ ). Topographical resolution is typically comparable to that of conventional AFM (i.e. of the order $\mathrm{nm}$ ) and images are similarly affected by tip-shape artefacts. The spatial resolution of the electrochemical measurement varies with working distance, as well as the precise tip geometry and mediator diffusion coefficient, but features in the 10 s of nm can usually be resolved.

Application of SECM-AFM is exemplified here by combined topographical-electrochemical imaging of exfoliated graphene, the electrochemistry of which is of considerable interest from the perspective of potential energy conversion and storage technologies. In this case a simple one-electron redox mediator was employed (ferrocenemethanol), for which the observed positive feedback currents were found to be highly non-uniform across the graphene flake (Figure 3c). Moreover, little correlation was observed between the measured Faradaic current and the thickness of the graphene (see regions $\mathrm{A}, \mathrm{B}$ and $\mathrm{C}$ in Figure $3 \mathrm{~d}$ ).
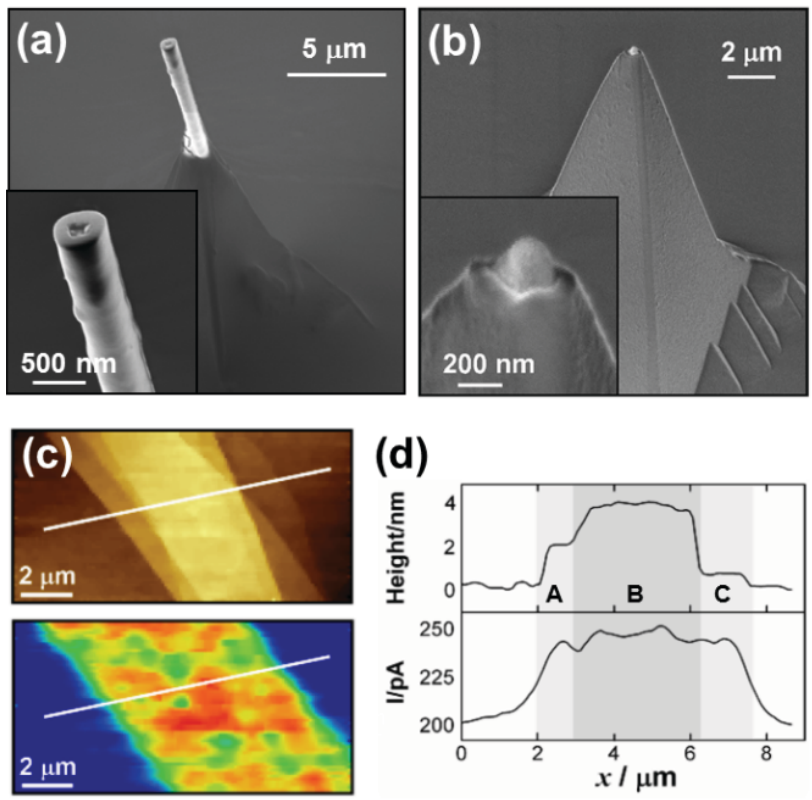

Figure 3. SEM images of (a) needle probe and (b) conical probe for SECM-AFM. (c) Topography (top) and Faradaic current (bottom) map of graphene flake imaged using conical probe. (d) Cross sections of height and current across graphene flake indicating three regions: (A) few-layer, (B) multi-layer and (C) single-layer graphene (adapted from [11] and [12]).

Whilst the sample was unbiased in this work, limiting the available information about electrokinetics, the high resolution topographical-electrochemical imaging highlighted the dominant effect of surface contamination that appears to block electron transfer in varying degrees across the surface.[12]

\subsection{SECM-SICM}

In SICM, an ionic current is passed through a small $(>50 \mathrm{~nm})$ aperture at the apex of a pulled glass capillary, and the magnitude of this current is sensitive to physical proximity to the surface and so can be used as a means to track sample topography. SICM in isolation is commonly employed as a non-contact technique for the imaging of soft biological systems, 
such as living cells, that necessitate measurement in electrolyte environments.[13]

The addition of a Faradaic electrode to the capillary enables the simultaneous acquisition of topography and electrochemical activity, in a similar fashion to SECMAFM, albeit with poorer spatial resolution in both channels by approximately a factor of ten. Integration of the Faradaic electrode can be achieved in various ways. We have focused on the dual barrel capillary approach developed by Takahashi et al.,[14] in which one barrel is filled with carbon to yield a solid nanoelectrode for Faradaic current measurement whilst the second barrel is left open to provide the SICM topography channel (Figure $4 \mathrm{a}$ ). These probes were shown to enable highresolution electrochemical imaging of model substrates as well as neuron cells.[14]

(a)

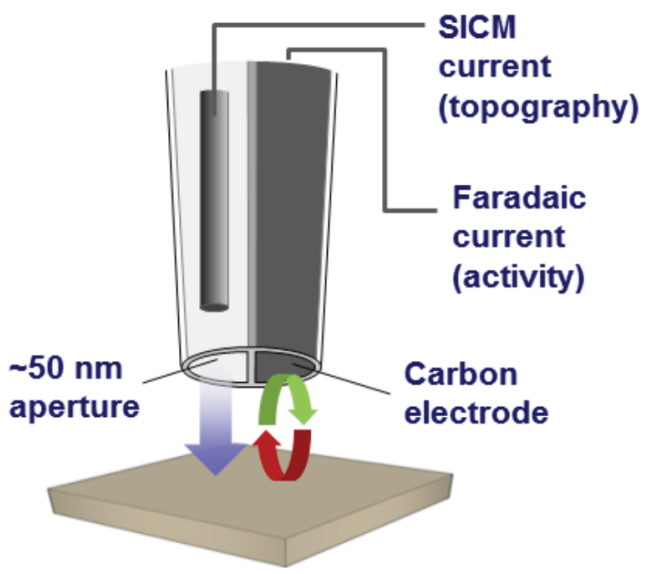

(b)
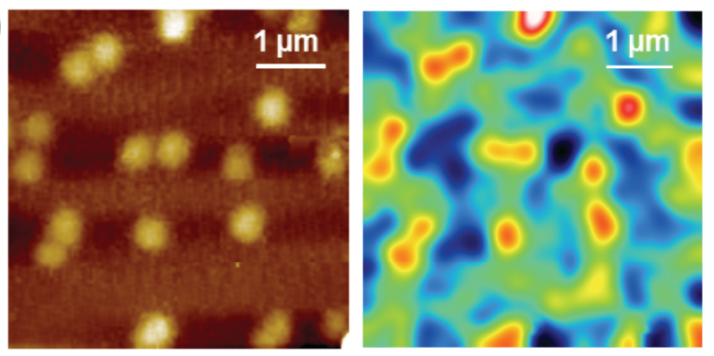

(c)
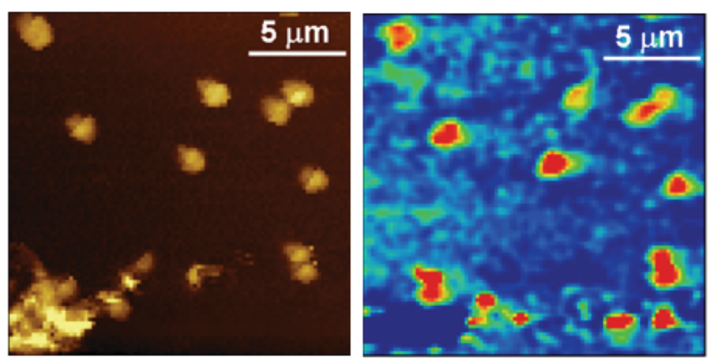

Figure 4. (a) Schematic depiction of dual barrelled capillary probes employed for SECM-SICM. (b) SECM-SICM images of platinum nanoparticle ensemble showing topography (left) and oxygen reduction current (right). Red colour indicates regions of most depleted oxygen due to the catalytic activity of the nanoparticles (adpated from [15]). (c) SECM-SICM images of gold nanoparticle ensemble showing topography (left) and hydrogen peroxide collection current (right). Red colour indicates highest concentration of hydrogen peroxide (adapted from [16]).
We recently extended the application of this approach to map the activity of platinum nanoparticle ensembles on glassy carbon surfaces.[15] As already discussed in the context of microarray screening, oxygen reduction is an important target reaction and so we employed the same competitive oxygen approach as above in which the tip senses oxygen depletion at the surface due to active electrocatalyst particles. This required making the probe more sensitive to oxygen reduction by depositing platinum on the carbon tip. As depicted in Figure 4b, the $\sim 100 \mathrm{~nm}$ diameter spherical nanoparticles are easily resolved in the topography channel and good contrast is observed in the SECM current, enabling assessment of qualitative oxygen reduction activity at the single particle level.

Taking this further we have implemented the same probes in a substrate generation - tip collection mode to detect the evolution of hydrogen peroxide during the oxygen reduction reaction.[16] This is an important element in the characterisation of oxygen reduction electrocatalysts since the liberation of hydrogen peroxide is not only an indication of the inefficient 2-electron reduction mechanism (as opposed to the complete 4-electron reduction to water), but also peroxide can have detrimental effects on fuel cell performance. SECM-SICM was applied in this mode to map hydrogen peroxide generation at gold nanoparticle ensembles, as shown in Figure 4c. Again, individual particles can be resolved in both the topographical image and the peroxide generation map. It was found in some cases that the amount of hydrogen peroxide generated did not correlate to the particle size (i.e. some similar sized particles exhibited quite different peroxide generation behaviour). This can be linked to significant differences in the shape and morphology of the particles imaged, that are at present beyond the resolution of this technique. Together with the aid of finite element modelling, it is envisaged that these advances could pave the way for more quantitative kinetic and mechanistic analysis at single nanoparticles within complex samples.

\section{Metrology considerations}

Given the widespread application of conventional SECM and the developments made in improving its spatial resolution, it is instructive to consider some metrological aspects of this technique.

The results reviewed in this paper have been largely qualitative, but quantitative SECM is possible and can be used to determine electrochemical kinetics as well as measure mass transport phenomena. This requires detailed knowledge of the tip geometry and dimensions in order to implement mathematical treatment of the diffusion equation under various conditions of electron transfer at the substrate. For simple micron-sized disk electrodes, measurement of the probe size and geometry is trivial, and rigorous theoretical analysis has been reported.[1] More complex probe architectures, such as 
those described for high-resolution imaging, require numerical modelling methods, but more challenging is the fact that smaller probes tend to be less regular and thus deviate considerably from model behaviour. Hence, a major requirement for more quantitative highresolution SECM is tighter control and consistency in the fabrication of nanoscopic probes. With smaller probes also comes the need to rigorously characterise the dimensions and geometry of every probe prior to use, especially due to issues related to electrode degradation or contamination, which can be very significant at this scale. As probes become smaller it will furthermore become necessary to replace finite element modelling with multiphysics simulation, in order to account for phenomena at a range of length scales.

The influence on probe movement during SECM measurements is an important consideration. Rapid scanning in any direction can introduce convection and, moreover, there is a significant risk that the probe electrochemistry is not at steady state, which can lead to misinterpretation of data. For quantitative approach curves (i.e. in the $z$-direction) slow scanning can be easily implemented, but doing so for mapping can be highly time-consuming. This can be particularly problematic if the sample or tip exhibit changing behaviour over time.

In terms of measurement uncertainties, the interpretation of absolute currents during electrochemical measurements can be problematic since electron transfer, as well as the associated mass transport, can be highly sensitive to the surface condition of the probe electrode. This can be alleviated by normalising tip current to the value measured in bulk solution, but this approach should be used cautiously for quantitative analysis, especially if currents deviate considerably from ideal values. Calibration of potentiostats as well as stage positioning hardware (step motors, piezos) is also necessary if the measurements demand a high degree of accuracy.

Finally, model test substrates are essential for validating mapping measurements although there is no formal standard at present. Commercially available or in-house fabricated patterned substrates are a common choice for low resolution imaging, but for high-resolution techniques durable samples with well-defined surface topography and sample uniformity at the nanometer scale are more difficult to produce.

\section{Conclusions}

Interfacial chemical activity is not a field that is commonly associated with metrology, but the need for advancing measurement science to understand chemical processes at complex surfaces continues to grow. Developments in SECM allow increasingly detailed analysis of surface processes with improving spatial resolution. With these improvements comes the necessity for better control over probe fabrication and reliable implementation in order for quantitative measurement and modelling to become routine.

\section{Acknowledgements}

This work was financially supported by the UK National Measurement System and the European Metrology Research Programme (EMRP, Ind 15 SurfChem). The EMRP is jointly funded by the EMRP participating countries within EURAMET and the European Union.

\section{References}

1. Scanning Electrochemical Microscopy, A.J. Bard, M.V. Mirkin (Eds.), CRC Press, (2012).

2. M.V. Mirkin, W. Nogala, J. Velmurugan, Y. Wang, Phys. Chem. Chem. Phys., 13, $21196-$ 21212 (2011).

3. S.C.S. Lai, J.V. Macpherson, P.R. Unwin, MRS Bull., 37, 668-674 (2012).

4. C. Kranz, Analyst, 139, 336-352 (2014).

5. M.A. O'Connell, A.J. Wain, Anal. Methods, (2015).

6. P.G. Nicholson, S. Zhou, G. Hinds, A.J. Wain, A. Turnbull, Electrochim. Acta, 54, 4525-4533 (2009).

7. A.J. Wain, Electrochem. Commun., 46, 9-12 (2014).

8. A.J. Wain, Electrochim. Acta, 92, 383-391 (2013).

9. W. Kylberg, A.J. Wain, F.A. Castro, J. Phys. Chem. C, 116, 17384-17392 (2012).

10. C.E. Jones, J.V. Macpherson, Z.H. Barber, R.E. Somekh, P.R. Unwin, Electrochem. Commun., 1, 55-60 (1999).

11. A.J. Wain, D. Cox, S. Zhou, A. Turnbull, Electrochem. Commun., 13, 78-81 (2011).

12. A.J. Wain, A.J. Pollard, C. Richter, Anal. Chem., 86, 5143-5149 (2014).

13. Y.E. Korchev, C.L. Bashford, M. Milovanovic, I. Vodyanoy, M.J. Lab, Biophys. J., 73, 653-658 (1997).

14. Y. Takahashi, A.I. Shevchuk, P. Novak, Y. Zhang, N. Ebejer, J.V. Macpherson, P.R. Unwin, A.J. Pollard, D. Roy, C.A. Clifford, H. Shiku, T. Matsue, D. Klenerman, Y.E. Korchev, Angew. Chem. Int. Ed., 50, 9638-9642 (2011).

15. M.A. O'Connell, A.J. Wain, Anal. Chem., 86, 12100-12107 (2014).

16. M.A. O'Connell, J.R. Lewis, A.J. Wain, Chem. Commun., 51, 10314-10317 (2015). 